\title{
Research of Fault Diagnosis Based on Sensitive Intrinsic Mode Function Selection of EEMD and Adaptive Stochastic Resonance
}

\author{
Zhixing Li and Boqiang Shi \\ School of Mechanical Engineering, University of Science and Technology Beijing, Beijing 100083, China \\ Correspondence should be addressed to Boqiang Shi; shiboqiang@ustb.edu.cn
}

Received 10 July 2016; Accepted 12 October 2016

Academic Editor: A. El Sinawi

Copyright (C) 2016 Z. Li and B. Shi. This is an open access article distributed under the Creative Commons Attribution License, which permits unrestricted use, distribution, and reproduction in any medium, provided the original work is properly cited.

\begin{abstract}
A novel methodology for the fault diagnosis of rolling bearing in strong background noise, based on sensitive intrinsic mode functions (IMFs) selection of ensemble empirical mode decomposition (EEMD) and adaptive stochastic resonance, is proposed. The original vibration signal is decomposed into a group of IMFs and a residual trend item by EEMD. Constructing weighted kurtosis index difference spectrum (WKIDS) to adaptively select sensitive IMFs, this method can overcome the shortcomings of the existing methods such as subjective choice or need to determine a threshold using the correlation coefficient. To further reduce noise and enhance weak characteristics, the adaptive stochastic resonance is employed to amplify each sensitive IMF. Then, the ensemble average is used to eliminate the stochastic noise. The simulation and rolling element bearing experiment with an inner fault are performed to validate the proposed method. The results show that the proposed method not only overcomes the difficulty of choosing sensitive IMFs, but also, combined with adaptive stochastic resonance, can better enhance the weak fault characteristics. Moreover, the proposed method is better than EEMD and adaptive stochastic resonance of each sensitive IMF, demonstrating the feasibility of the proposed method in highly noisy environments.
\end{abstract}

\section{Introduction}

Rolling bearings are widely used in large or small mechanical equipment $[1,2]$. In severe cases when rolling bearings early break down during serving the machines, the machines can be damaged. In fact, the unpredictable failures may cause serious damage to machinery and equipment, so the extraction of early defects is very important to ensure the reliable operation of the machinery [3]. It is difficult for the vibration signal of the rolling bearing which is nonstationary and strongly modulated to detect weak fault characteristics under strong background noise [4]. The traditional signal processing methods are mainly to reduce or eliminate the noise. Empirical mode decomposition method is an effective tool for processing the complex nonstationary signal, but it has a disadvantage which is the mode mixing [5]. Later, $\mathrm{Wu}$ and Huang [6] have proposed ensemble empirical mode decomposition (EEMD) by introducing the additional noise, and this method has overcome the shortcoming of the traditional empirical mode decomposition to some extent.
The complex nonstationary vibration signal can be decomposed into many intrinsic mode functions (IMFs), and these functions can reflect the real physical information in the vibration signals. However, some of these IMFs are useless or interfering components and the others are sensitive components to fault characteristics. Many researchers extracted the fault features by selecting the effective and sensitive IMFs to the mechanical faults, thereby eliminating the noise components or the unrelated-fault components. Hu et al. $[7,8]$ proposed a method of selecting the sensitive IMFs by using the largest kurtosis index; many kurtosis values may be greater than 3; the maximum kurtosis value corresponding to IMFs may lose part of the fault information. Tan et al. [9] proposed a method to select sensitive IMFs by using correlation coefficient, but the method needs to determine a threshold to select sensitive IMFs components. According to different signals, the threshold value required to be adjusted artificially. In order to overcome the above shortcomings, this paper proposed a method that constructs weighted kurtosis index difference spectrum for selecting effective 
and sensitive IMFs to the faults. However, in the strong background noise, even if the effective IMFs are obtained, the fault feature is still very weak, which cannot identify the fault characteristics effectively. For the fault diagnosis of weak signal, stochastic resonance is widely used for its unique advantages. Stochastic resonance was first proposed in 1981 in the study of Earth's glaciers and it has attracted a lot of attention in the past thirty years, especially in denoising and signal enhancement [10]. When the useful signal and the noise are mixed, the traditional denoising methods will damage the useful signal and lose the vibration features. At the same time, the traditional methods cannot extract the fault features where the signal-to-noise ratio is less than $-15 \mathrm{~dB}$. Stochastic resonance is used to enhance the weak fault characteristics and reduce the noise, but the performance of stochastic resonance methods is mostly decided by system parameters of the potential function of stochastic resonance [11]. The existing stochastic resonance methods ignore the interactive effect between parameters through subjectively selecting parameters or optimizing only one parameter [12]. Zhang et al. [13] extended the frequency range of stochastic resonance by scaling transform and applied it to the fault characteristic detection of bearing outer race. In addition, multiple parameters of stochastic resonance system need to be optimized to ensure the effectiveness of stochastic resonance. This paper quoted the method using the ant colony algorithm to parallelly optimize multiple parameters of stochastic resonance [14-16]. Therefore, combining the advantages of EEMD reduced noise and stochastic resonance enhanced weak fault characteristics, this paper proposes a fault diagnosis method based on sensitive IMFs selection of EEMD and adaptive stochastic resonance. Firstly, the original vibration signal is decomposed by EEMD. The modulated vibration signal is demodulated effectively and the frequency features of vibration signal are decomposed into several IMFs components. These obtained IMFs components are selected by weighted kurtosis index difference spectrum (WKIDS). Finally, the selected sensitive IMFs are input into the stochastic resonance system. In order to further reduce noise, the enhanced sensitive IMFs via stochastic resonance are ensemble-averaged. Finally, the weak fault characteristics are realized in the strong background noise, and the validity of the sensitive IMFs selection of EEMD and adaptive stochastic resonance method is verified by simulation and experiment.

The other parts of this paper are as follows. Section 2 introduces the basic principles of EEMD and stochastic resonance. Section 3 describes the method for the sensitive IMFs selection of EEMD and adaptive stochastic resonance. Constructing WKIDS, the selected IMFs are input into adaptive stochastic resonance. To reduce noise further and enhance the weak signal and the adaptive stochastic resonance of each sensitive IMF, ensemble average is used to eliminate the stochastic noise. The effectiveness of the proposed method is verified by the periodic pulse signal simulation in Section 4 . The inner ring fault of bearing is detected by the proposed method in Section 5. Finally, the conclusion is given in Section 6.

\section{Principle Analyses}

2.1. Ensemble Empirical Mode Decomposition. Huang et al. $[17,18]$ proposed empirical mode decomposition to decompose any complicated signal, which has been widely studied and applied in the fault diagnosis field. However, the empirical mode decomposition method still needs improvement in some areas. An important limitation is the mode mixing problem. Recently, Wu and Huang [6] proposed a new method (EEMD) to solve the mode mixing which can restore the nature of the original signal. It is known to be a great improvement of empirical mode decomposition and is widely used in the machinery field [19].

The principle of EEMD method used the Gaussian white noise with a uniform frequency distribution of the statistical characteristics; when the signal is added to the Gaussian white noise, the signal will be continued at different scales. In order to reduce the degree of mode mixing, the specific decomposition steps and principles are shown as follows.

Step 1. In the original signal, $x(t)$ is added to the Gaussian white noise $n_{i}(t)$ with the amplitude mean value of 0 , and the standard deviation is constant. Hence,

$$
x_{i}(t)=x(t)+n_{i}(t) .
$$

In the formula, $x_{i}(t)$ represents the Gaussian white noise signal that is added for $i$ times, and the range size of the Gaussian white noise will directly affect the signal EEMD to avoid the decomposition effect of the mode mixing.

Step 2. $x_{i}(t)$ is decomposed by empirical mode decomposition and hence we get the IMF component $c_{i j}(t)$ and the remainder $r(t) . c_{i j}(t)$ indicates that, after the $i$ times, Gaussian white noise is added and the $j$ th component is obtained.

Step 3. Repeat Steps 1 and $2 N$ times. Using the principle of uncorrelated random sequence with the statistical mean value of 0 , the IMF corresponding to the above will have total average operation and will eliminate the influence on IMF while the white Gaussian noise is added repeatedly. Finally, the IMFs are obtained after EEMD:

$$
c_{j}(t)=\frac{1}{N} \sum_{i=1}^{N} c_{i j} .
$$

In formula $(2), c_{j}(t)$ represents the notion that the $j$ th IMF component is obtained from the EEMD of the original signal. The greater the value of $N$ is, the more the IMFs of Gaussian white noise tend to be 0 . So, the results of EEMD are as follows:

$$
x(t)=\sum_{j} c_{j}(t)+r(t) .
$$

$r(t)$ is the final residual component and represents the average trend of the signal. The EEMD method can decompose any signal into the sum of a set of IMFs and a residual component. The intrinsic mode component $c_{j}(t)(j=1,2, \ldots)$ represents a component of a signal from high to low frequency range. 


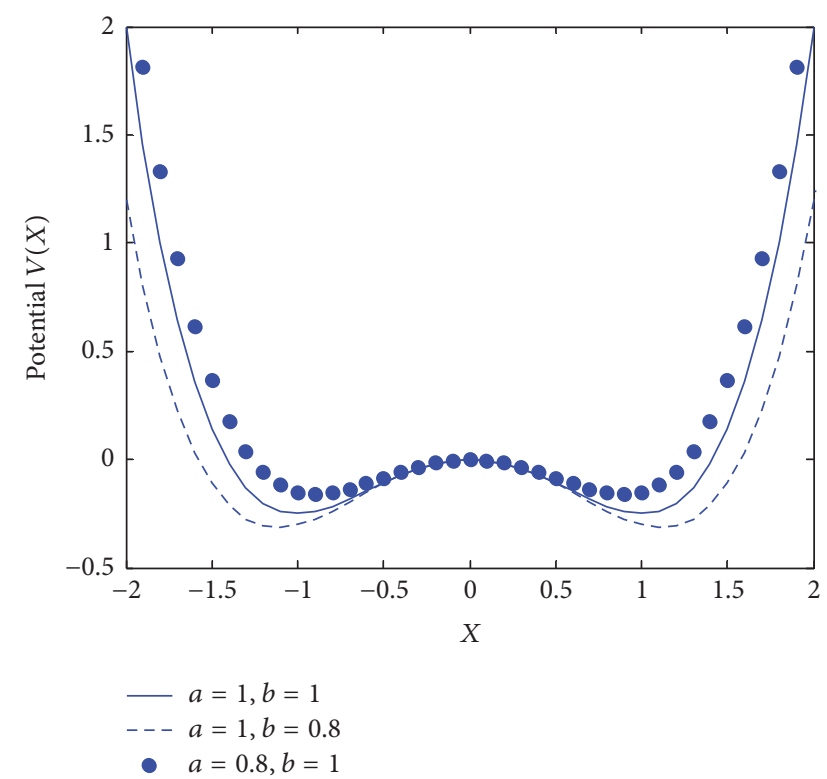

FIGURE 1: The potential function in different cases.

Each frequency band contains different frequency components and may vary with the change of the vibration signal $x(t)$.

2.2. Model of Stochastic Resonance. Nowadays, the bistable system is widely studied which is driven by the Gaussian white noise and external periodic signal. This bistable system can be defined as follows:

$$
\begin{aligned}
\frac{d x}{d t} & =\frac{-\partial V(x)}{\partial x}=S(t)+N(t), \\
\langle N(t)\rangle & =0 \\
\langle N(t), N(0)\rangle & =2 D \delta(t),
\end{aligned}
$$

where $V(x)$ is potential function of nonlinear bistable system

$$
V(x)=-\frac{a}{2} x^{2}+\frac{b}{4} x^{4}, \quad a>0, b>0,
$$

where periodic external force $S(t)=A \cos (w t)$ and $N(t)$ is Gaussian white noise in which the intensity is $D$ and the mean value is 0 . Accordingly, we can obtain (6) by operating (4) and (5):

$$
\frac{d x}{d t}=a x-b x^{3}+S(t)+N(t)
$$

where $x$ is the output signal of stochastic resonance system and $a$ and $b$ are the parameters of stochastic resonance system; meanwhile, the system parameters satisfy $a>0$, $b>0$. This equation indicates that, in two potential wells, the overdamped motion of the particle is driven by periodic signal and noise. The potential function $V(x)$ has two stable fixed points at $x= \pm \sqrt{a / b}$, the height of potential barrier is $\Delta V(x)=a^{2} / 4 b$, and the barrier top is located at $x=0$. To be specific, we choose $a=b=1 ; a=0.8, b=1 ; a=1, b=$ 0.8 . Figure 1 shows the different states of potential functions
$V(x)$ at different stochastic resonance system parameters $a$ and $b$. It is clear that two potential wells are symmetric and separated by a barrier with the height changes with different system parameters $a$ and $b$, so as to achieve the best stochastic resonance $[20,21]$. Hence, we can achieve the aim of detecting weak signals successfully by adjusting the system parameters $[22,23]$.

\section{The Proposed Diagnosis Method Based on Sensitive IMFs Selection and Stochastic Resonance}

3.1. Selection of Sensitive IMFs. Kurtosis index is very sensitive to the fault characteristic in the strong background noise. Generally, kurtosis index is used for measuring the impulsive characteristics of the vibration signals. Kurtosis index formula is written as [24]

$$
K=\frac{E\left(r^{4}\right)}{E^{2}\left(r^{2}\right)}=\frac{(1 / T) \sum_{t=1}^{T}\left(r_{t}-\bar{r}\right)^{4}}{\sigma^{4}} .
$$

In the formula, $r=\left(r_{1}, r_{2}, \ldots, r_{t}, \ldots, r_{T}\right)$ is the signal sequence, $T$ is the signal length, $\bar{r}$ is the mean value of the signal, and $\sigma$ is the standard deviation of the signal

$$
\sigma=\sqrt{\frac{1}{T} \sum_{t=1}^{T}\left(r_{t}-\bar{r}\right)^{2}} .
$$

Equation (7) turns to

$$
\begin{aligned}
K & =\frac{T^{2} \sum_{t=1}^{T}\left(r_{t}-\bar{r}\right)^{4}}{T\left(\sum_{t=1}^{T}\left(r_{t}-\bar{r}\right)^{2}\right)^{2}}=\frac{T M_{1}}{M_{2}}, \\
M_{1} & =\sum_{t=1}^{T}\left(r_{t}-\bar{r}\right)^{4}, \\
M_{2} & =\left(\sum_{t=1}^{T}\left(r_{t}-\bar{r}\right)^{2}\right)^{2}, \\
M_{2}-M_{1} & =2 \sum_{t=1}^{T}\left(r_{t}-\bar{r}\right)^{2} \sum_{i=1, i \neq t}^{T}\left(r_{i}-\bar{r}\right)^{2}=P(r) .
\end{aligned}
$$

Because $\bar{r}$ is the mean value of the signal, when the pulse signal increases or decreases, it will have little influence on $\bar{r}$. When the pulse signal increases, $P(r)$ increases and thus $K$ decreases. Similarly, when the pulse signal decreases, $K$ increases. When there are multiple pulse signals in the strong background noise, one or more of the pulse signals are hidden in the noise that may not be detected; $K$ does not decrease but increases. The loss of the pulse signal is called "leakage peak phenomenon." Therefore, kurtosis index curve cannot reflect all the information of IMFs. 
$C$ represents the correlation of the two signals. The more the similarity they have, the greater the value of $C$. The crosscorrelation coefficient between signals $x(t)$ and $y(t), C$, is defined as

$$
C=\frac{\sum_{n=0}^{T}(x(t)-\bar{x})(y(t)-\bar{y})}{\left[\sum_{n=0}^{T}(x(t)-\bar{x}) \sum_{n=0}^{T}(y(t)-\bar{y})\right]^{1 / 2}} .
$$

Because the fault vibration signal $x(t)$ is decomposed into IMFs components by EEMD, each IMF component $x_{\mathrm{IMF}}$ is similar to $x(t)$, and their cross-correlation coefficient is $C_{1} \cdot x_{\text {IMF }}$ is also similar to normal working condition signal $x_{\text {nor }}(t)$, and each IMF component and normal working condition signal's cross-correlation coefficient is $C_{2} . C_{1}$ and $\mathrm{C}_{2}$ are defined as

$$
\begin{aligned}
& C_{1}=\frac{\sum_{n=0}^{T}(x(t)-\bar{x})\left(x_{\mathrm{IMF}}(t)-\bar{x}_{\mathrm{IMF}}\right)}{\left[\sum_{n=0}^{T}(x(t)-\bar{x}) \sum_{n=0}^{T}\left(x_{\mathrm{IMF}}(t)-\bar{x}_{\mathrm{IMF}}\right)\right]^{1 / 2}}, \\
& C_{2} \\
& =\frac{\sum_{n=0}^{T}\left(x_{\mathrm{nor}}(t)-\bar{x}_{\mathrm{nor}}\right)\left(x_{\mathrm{IMF}}(t)-\bar{x}_{\mathrm{IMF}}\right)}{\left[\sum_{n=0}^{T}\left(x_{\mathrm{nor}}(t)-\bar{x}_{\mathrm{nor}}\right) \sum_{n=0}^{T}\left(x_{\mathrm{IMF}}(t)-\bar{x}_{\mathrm{IMF}}\right)\right]^{1 / 2}} .
\end{aligned}
$$

Combined with the two cross-correlation coefficients, a new cross-correlation coefficient $C_{K}=C_{1}-C_{2}$ is obtained which is associated with fault. $C_{K}$ is considered not only the similarity between each IMF and the normal signal, but also the similarity of the fault vibration signal. Because of the absolute value $C_{K}$ which is a real number and is no more than 1 , it has the potential to be the weight.

In the strong background noise and multiple pulse signals, one or more pulse signals are hidden by the noise, and $K$ increases. However, the similarity of pulse signals associated with fault is reduced; that is, $C_{K}$ decreases, and the decrease of $C_{K}$ corrects the increase of $K$. Therefore, this paper uses $C_{K}$ as the weight of $K$ to construct weighted kurtosis index $K_{W}$ which is defined as

$$
K_{W}=C_{K} K
$$

The weighted kurtosis index can not only prevent the loss of vibration signal, but also ensure the similarity of vibration signal. Hence, the weighted kurtosis index curve reflects all the information of IMFs. Let the sequence of IMFs corresponding to the weighted kurtosis index defined as $S=\left(K_{W}^{1}, K_{W}^{2}, \ldots, K_{W}^{q}\right)$ lead to the differential spectrum. The equation is $b^{l}=K_{W}^{l}-K_{W}^{l+1}(l=1,2, \ldots, q-1)$. Then, all $b^{l}$ forming a sequence is

$$
B=\left(b^{1}, b^{2}, \ldots, b^{q-1}\right)
$$

$B$ is named weighted kurtosis index difference spectrum (WKIDS). Difference spectrum is used for describing the changes of the adjacent weighted kurtosis index. When the difference between adjacent weighted kurtosis indexes is large relatively, there is a maximum mutation point $b_{k}$ in the whole difference spectrum corresponding to the weighted kurtosis index sequence at $k . b_{k}$ reflects the biggest difference between the weighted kurtosis index properties. In other words, signals generate a mutation between the useful IMFs signals and the noise signals at $b_{k}$. Therefore, a method of selecting effective IMFs signals can be proposed. In the weighted kurtosis index sequence, the points before the mutation point including it, $k$ weighted kurtosis index corresponds to useful IMFs. After the mutation point, the weighted kurtosis index corresponds to useless IMFs. In this sense, the $k$ sensitive IMFs are selected by WKIDS.

3.2. Adaptive Stochastic Resonance. Because the fault characteristics of mechanical equipment are hidden in strong background noise, a large amount of background noise greatly reduces the signal-to-noise ratio. Although $k$ sensitive IMFs are selected by KWIDS method, it is difficult to extract the fault characteristics from them.

Therefore, sensitive IMFs of EEMD are further enhanced by adaptive stochastic resonance method. The traditional stochastic resonance is only applicable to small parameter signal (signal amplitude $A \leq 1$, noise intensity $D \leq 1$, and signal frequency $f \leq 1$ ). Therefore, the sensitive IMFs before inputting into stochastic resonance system are processed by frequency-shifted and scaling transform to satisfy the small parameter conditions. The principle of the method is to design a frequency compression scale, and the frequency of sensitive IMFs is compressed to determine the calculation step size. This way, the IMFs meet the requirements of small parameters. The system parameters $a$ and $b$ of stochastic resonance have a decisive effect on its performance [25]. In this paper, a good global optimization ability of ant colony algorithm is used to search the optimal parameters of stochastic resonance. The optimal combination of parameters $a$ and $b$ is brought into stochastic resonance and the signal-tonoise ratio of each sensitive IMF is calculated as the objection function of ant colony algorithm. Thus, the enhanced $k$ IMFs are obtained. In order to reduce the stochastic interference in each enhanced IMF, each enhanced IMF was ensembleaveraged, eliminating the effects of noise in multiple IMFs. So, the final result is defined as

$$
b(t)=\frac{1}{k} \sum_{i=1}^{k} b_{i}
$$

In formula (17), $b_{i}$ represents the $i$ th effective IMF component.

In the paper, the flowchart of the proposed method is shown in Figure 2. The procedure of the method is described as follows.

(1) The bearing vibration signals are decomposed by EEMD, obtaining $N$ IMFs and a trend term.

(2) A method of WKIDS is proposed; $k$ sensitive IMFs are selected by WKIDS, which are treated as the input of stochastic resonance system.

(3) Initiate the parameters of ant colony algorithm and select evaluation function of stochastic resonance. Here, the evaluation function is set as the signal-to-noise ratio of output signal of stochastic resonance system. 


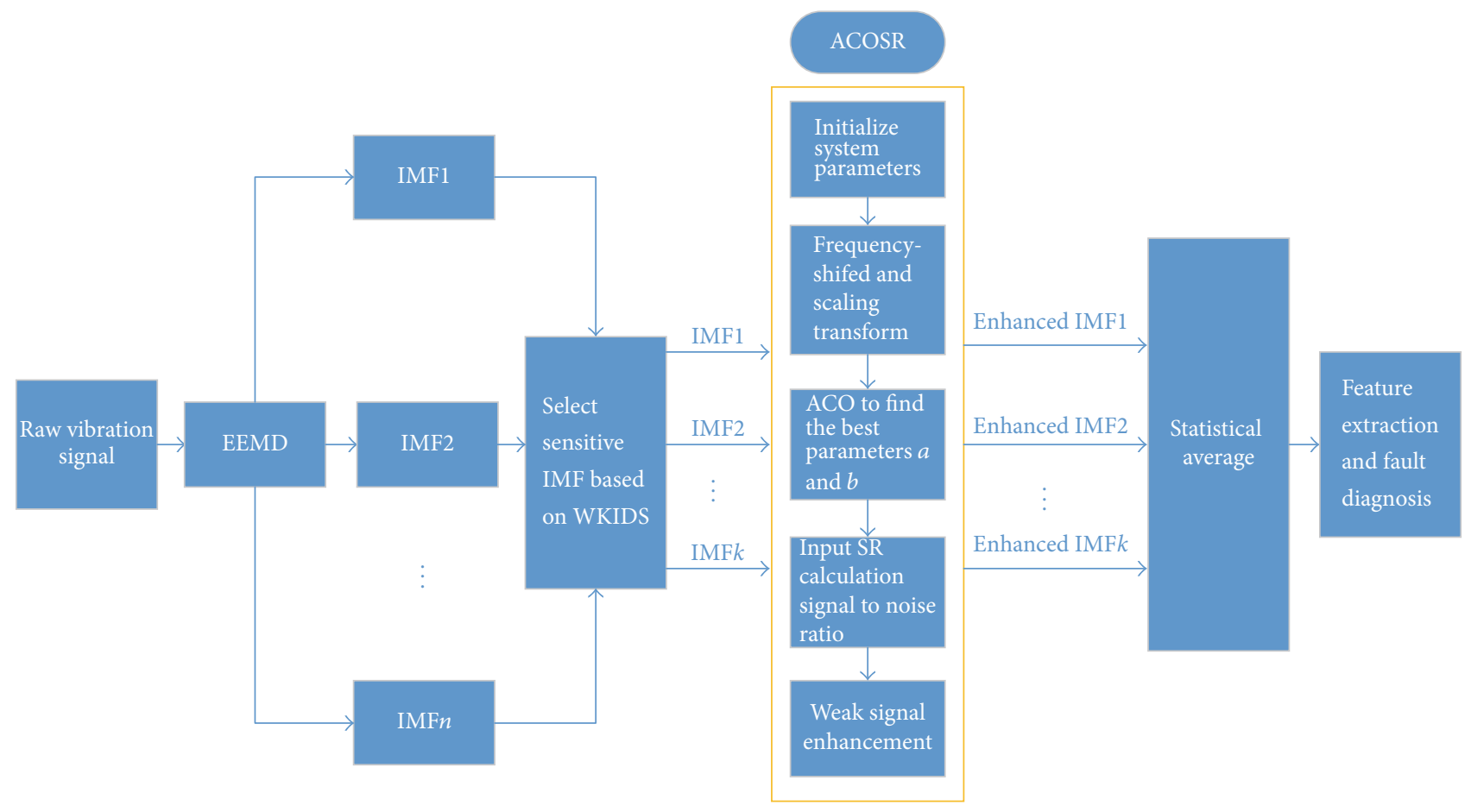

FIGURE 2: The flowchart of the proposed method.

(4) The $k$ sensitive IMFs are initially processed by frequency-shifted and scaling transform to meet the requirements of small parameters.

(5) Employ ant colony algorithm to optimize the two parameters $a$ and $b$ of stochastic resonance and thereby obtain the optimal combination of $a$ and $b$. The signal-to-noise ratio of $k$ sensitive IMFs is calculated as an evaluation function and further $k$ enhanced IMFs are obtained.

(6) The $k$ enhanced IMFs are ensemble-averaged to get the final enhanced signal, and the fault characteristic frequency is extracted from it.

\section{Simulated Verification}

In order to validate the effectiveness of the method proposed, periodic pulse of the bearing fault simulation signal is employed in [26]. The sampling frequency of the periodic pulse signal is $10 \mathrm{kHz}$. The characteristic frequency is $50 \mathrm{~Hz}$, and the sampling time is $1 \mathrm{~s}$. Time-domain waveform and frequency spectrum of the periodic pulse signal are shown in Figure 3.

The noise intensity with Gaussian white noise of 0.5 is added to the periodic pulse signal in order to simulate the operating background of rotating machinery. It is shown in Figure 4(a) that the periodic pulse signal is submerged in strong background noise. Therefore, the simulated signal has certain practical significance.

Due to the fact that the periodic pulse signal is hidden in the strong noise, we cannot obtain the periodic pulse characteristics from the time-domain waveform in Figure 4(a) and the corresponding frequency spectrum in Figure 4(b).

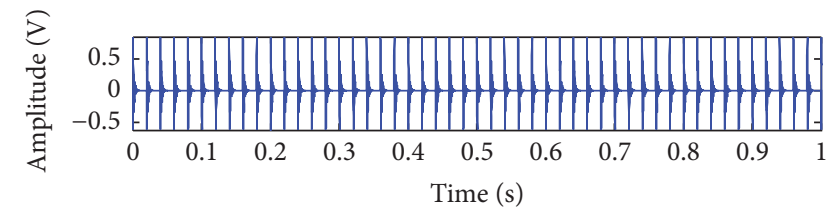

(a)

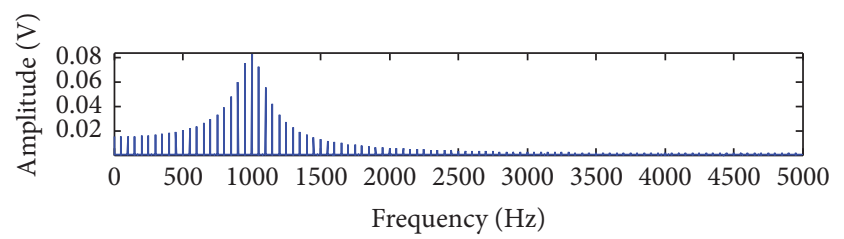

(b)

FIGURE 3: The periodic pulse signal: (a) time-domain waveform; (b) frequency spectrum.

We know that the envelope spectrum has a good effect on extracting the fault feature, but the fault characteristics are still hidden in Figure 4(c). Therefore, we conclude that the envelope spectrum cannot extract the characteristic frequency of the noisy signal in the strong background noise.

In order to obtain the characteristic frequency in strong background noise, the original signal is decomposed by EEMD, and then 13 IMFs and a residual trend item are obtained which are shown in Figure 5(a). IMF1 IMF6 are the high and low frequency components, IMF7 IMF9 are the low frequency components, and IMF10 IMF13 are the very low frequency components so that the characteristic 


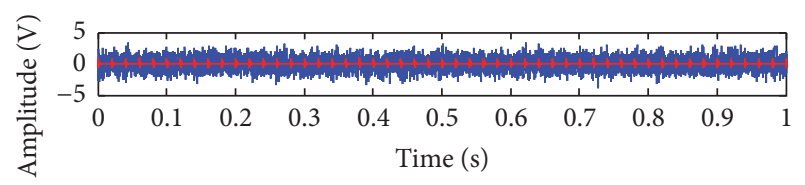

(a)

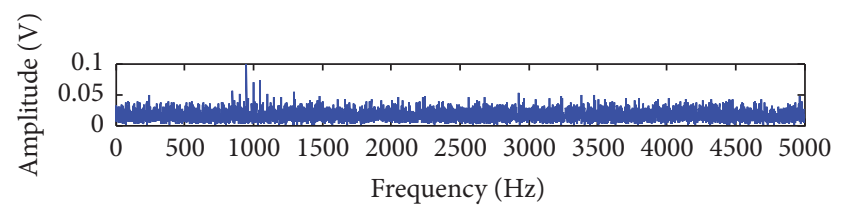

(b)

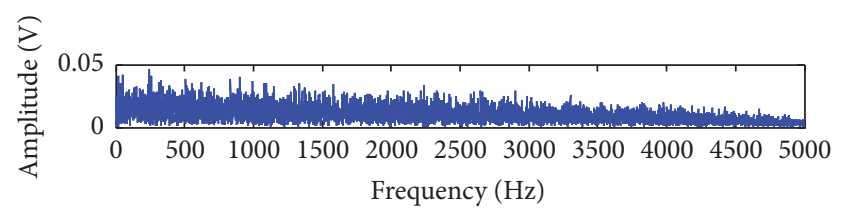

(c)

FIgURE 4: The noisy signal: (a) time-domain waveform; (b) frequency spectrum; (c) envelope spectrum.

frequency cannot be seen. The periodic signals cannot be clearly distinguished from the envelope spectrum in Figure 5(b). We use the method of KWIDS to select the sensitive IMFs. Firstly, according to (9), we can get the kurtosis index curves in Figure 6(a). From the graph, multiple kurtosis index values are greater than 3 which may cause the loss of some characteristics. Hence, the weighted kurtosis index is constructed by the cross-correlation coefficient to get all the fault information of the IMFs and the difference spectrum curve by formula (17) in Figure 6(b). Difference spectrum corresponding to IMFs is shown in Table 1, with IMF4 of 0.07206 and the maximum mutation point. Therefore, IMF1 IMF4 reflect the fault characteristics of the raw vibration signal and they are the sensitive IMFs input of adaptive stochastic resonance method.

According to frequency-shifted scaling transform method, the pass frequency and cutoff frequency of the high pass filter are $48 \mathrm{~Hz}$ and $40 \mathrm{~Hz}$, respectively. The modulation frequency is $40 \mathrm{~Hz}$ and the variable scale compression rate is 400 . The target frequency is compressed to $(50-40) / 400=0.025 \leq 1 \mathrm{~Hz}$ which meets the requirements of small parameters. IMF1 IMF4 input signals are input into stochastic resonance system, and two system parameters $a$ and $b$ of stochastic resonance are optimized by ant colony algorithm. Find the optimal parameter combination of adaptive stochastic resonance system in the range of $0<a<$ 10 and $0<b<10$, and the output signal-to-noise ratios of IMF1 IMF4 are 20.3957, 20.1430, 21.4371, and 18.5379, respectively, which is an evaluation function in Table 2.

The time-domain and the frequency spectrum of the IMF1 IMF4 components input into adaptive stochastic resonance are shown in Figures 7 10.

Because the adaptive stochastic resonance can enhance the weak fault characteristics buried in noise, the feature frequency of the component signal can be highlighted. The
TABLE 1: The difference spectrum of the simulation signal corresponding to IMFs.

\begin{tabular}{lc}
\hline IMF & Difference spectrum \\
\hline IMF1 & 0.01753 \\
IMF2 & 0.04468 \\
IMF3 & 0.01653 \\
IMF4 & 0.07206 \\
IMF5 & 0.001666 \\
IMF6 & 0.000916 \\
IMF7 & $9.036 e-5$ \\
IMF8 & 0.002096 \\
IMF9 & 0.000223 \\
IMF10 & 0.000174 \\
IMF11 & 0.000633 \\
IMF12 & $4.177 e-5$ \\
IMF13 & 0.000682 \\
\hline
\end{tabular}

adaptive stochastic resonance frequency spectrums of IMF1 IMF3 all have maximum value of $50 \mathrm{~Hz}$ in Figures 7(b), 8(b), and $9(\mathrm{~b})$, which are consistent with the actual situation, and thus they have noise interference, where the noise interference of IMF2 is especially serious. In Figure 10, due to the strong noise of the interference, the maximum spectral peak is $51.67 \mathrm{~Hz}$, instead of the target frequency. In order to reduce noise interference, overall average operation is performed on IMF1 IMF4, and the result is shown in Figure 11.

In Figure 11(b), the highest amplitude frequency is $50 \mathrm{~Hz}$, which is the same as the characteristic frequency, and the period of vibration signal is very obvious. In addition, the noise is largely eliminated by using stochastic resonance and statistical average. Compared with EEMD in Figure 5(b) and stochastic resonance of each IMF component in Figures 7 (b), 8(b), 9(b), and 10(b), it is obviously superior to all the above. In other words, the EEMD reduces noise but does not extract the features of the fault. Stochastic resonance of each IMF component is used to enhance the weak fault characteristics and this effect is not so perfect. In order to reduce noise further, the ensemble average is used for four enhanced IMFs. Finally, the fault characteristic frequency is extracted effectively and the peak is much higher than the surrounding noise. Therefore, we verified the validity of fault diagnosis method of sensitive IMFs selection of EEMD and adaptive stochastic resonance.

\section{Experimental Demonstration}

The equipment used in the experiment is a bearing fault test platform made by American spectra quest manufacturing company as shown in Figure 12. In the test, we used ZonicBook/618E type test system and the ER-10K cylindrical roller bearings as the failure bearing. The vibration signal is collected by the acceleration sensor which is mounted on the bearing seat. The cylindrical roller bearing geometry sizes are $n=8, D=33.5 \mathrm{~mm}, d=7.9375 \mathrm{~mm}$, and $\alpha=0^{\circ}$. In the test, sampling frequency is $5120 \mathrm{~Hz}$ and the speed of the shaft is $2100 \mathrm{r} / \mathrm{min}$. 


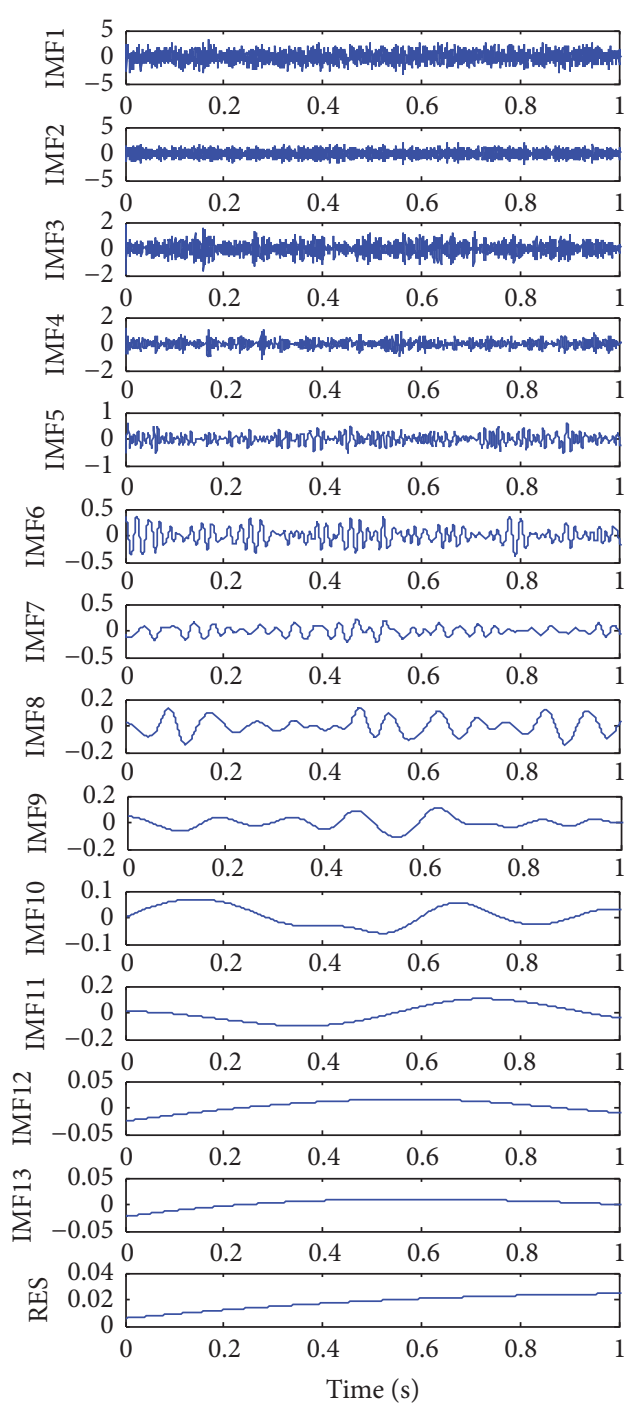

(a)

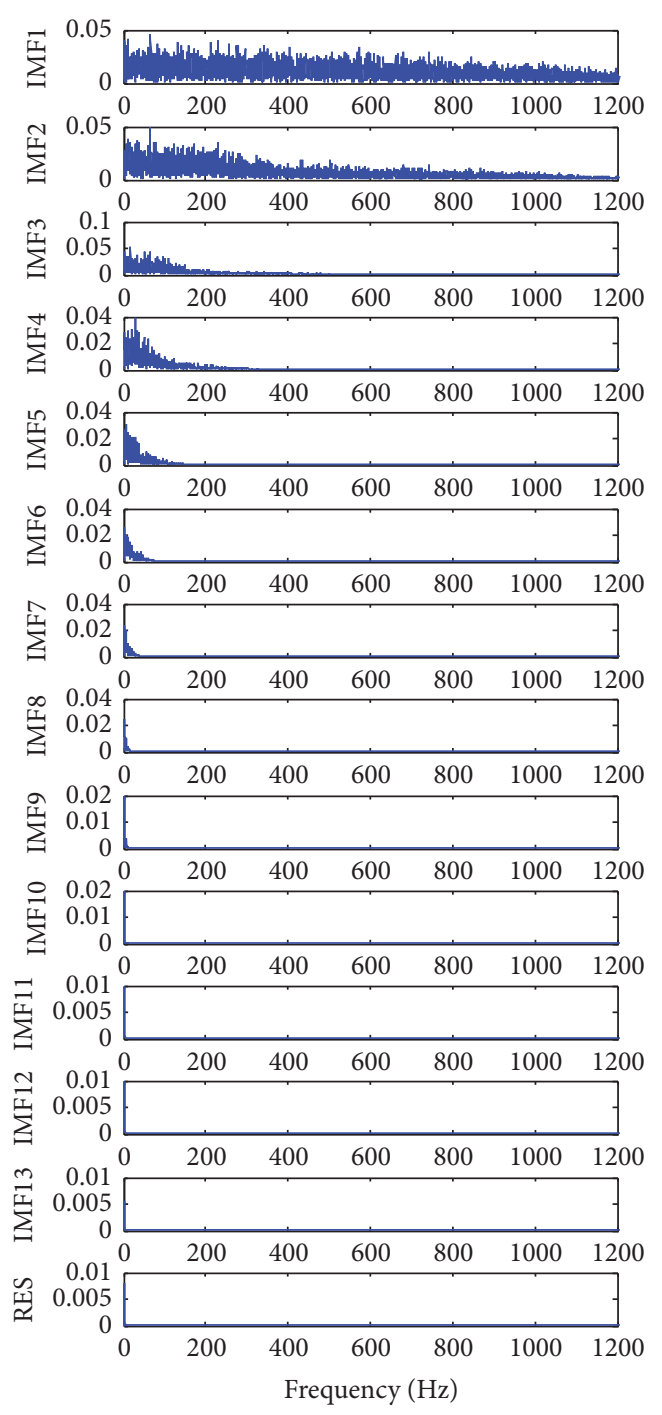

(b)

FIGURE 5: EEMD: (a) time-domain waveform; (b) envelope spectrum.

TABLE 2: The optimal parameter combination of adaptive stochastic resonance of IMF1 IMF4.

\begin{tabular}{lcccc}
\hline Parameter & IMF1 & IMF2 & IMF3 & \\
\hline$a$ & 0.2312 & 0.1187 & 0.1035 & IMF4 \\
$b$ & 9.7880 & 0.1378 & 0.543 & 10.0840 \\
Signal-to-noise ratio & 20.3957 & 20.1430 & 21.4371 & 18.5379 \\
\hline
\end{tabular}

According to fault characteristic frequency theory about the rolling bearings, we can know that the inner race fault frequency is $173.25 \mathrm{~Hz}$. Time-domain waveform of the bearing inner race is shown in Figure 13(a). Due to the fault signals being very weak in the strong background noise, it is not easy to find the fault characteristic frequency in the frequency spectrum and envelope spectrum that are shown in Figures 13(b) and 13(c). Therefore, we cannot determine whether the bearing inner race has a fault.

According to the method proposed in this paper, firstly, the raw vibration signal is decomposed by EEMD in which the time-domain waveform of IMF1 IMF4 is shown in Figure 14(a). The characteristic component of the bearing inner race fault is completely hidden in the background noise with a low signal-to-noise ratio, which cannot detect obvious spectral peaks in the frequency spectrum of IMF1 IMF4 in Figure 14(b), and the fault features cannot be extracted. Therefore, the proposed method is used to select the sensitive IMFs of EEMD and adaptive stochastic resonance.

In order to obtain the sensitive IMFs, constructing KWDIS according to (9) and kurtosis index curve is shown in Figure 15(a). The kurtosis index curve illustrates multiple 


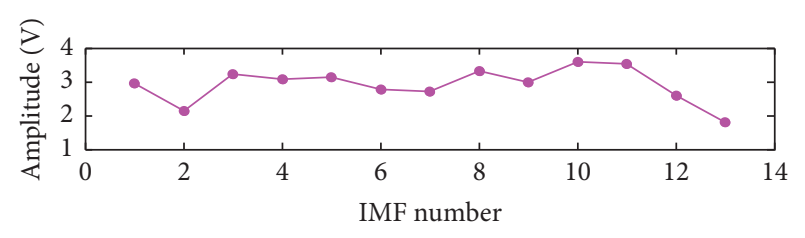

- Kurtosis index

(a)

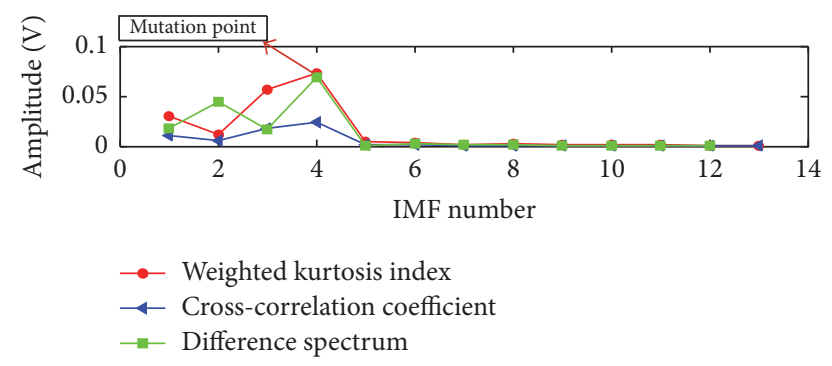

(b)

FIGURE 6: WKIDS of the inner race of the bearing: (a) kurtosis index; (b) weighted kurtosis index, cross-correlation coefficient, and difference spectrum.

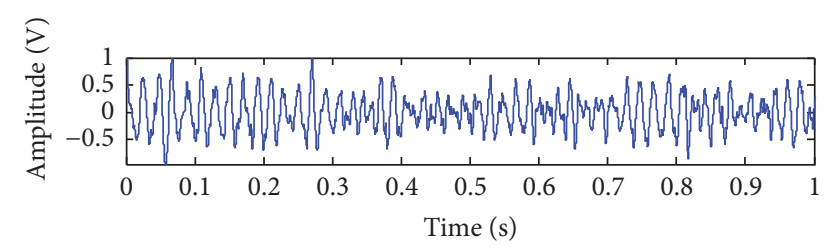

(a)

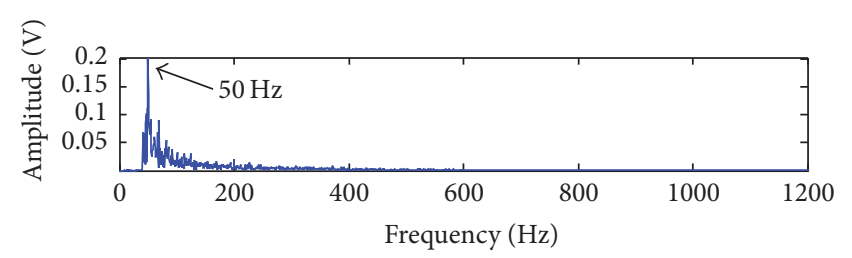

(b)

FIGURE 7: Optimal output signal of IMF1: (a) time-domain waveform; (b) frequency spectrum.

kurtosis index values greater than 3 which indicate that the extracted bearing inner race fault is distorted. Hence, the weighted kurtosis index is constructed by the crosscorrelation coefficient to get all bearing inner race faults information and difference spectrum by (17) in Figure 15(b). Difference spectrums corresponding to IMFs are shown in Table 3, with IMF3 of 0.9087 and the maximum mutation point. Therefore, IMF1 IMF3 reflect the fault characteristics of the bearing inner race and they were the sensitive IMFs input as adaptive stochastic resonance signals.

The selected IMF1 IMF3 are input into the adaptive stochastic resonance. Due to the fact that the target frequency of the bearing inner race fault is $173.25 \mathrm{~Hz}$, in the frequencyshifted and scaling transform pretreatment method, the pass frequency and cutoff frequency of the high pass filter are $170 \mathrm{~Hz}$ and $165 \mathrm{~Hz}$, respectively. The modulation frequency

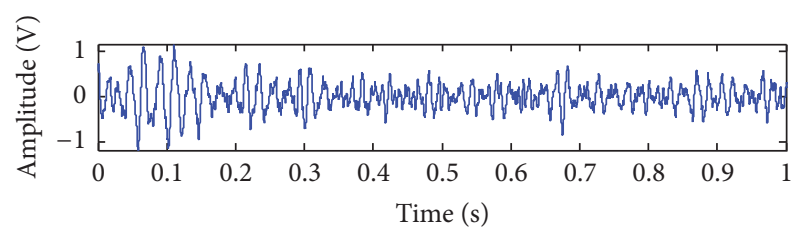

(a)

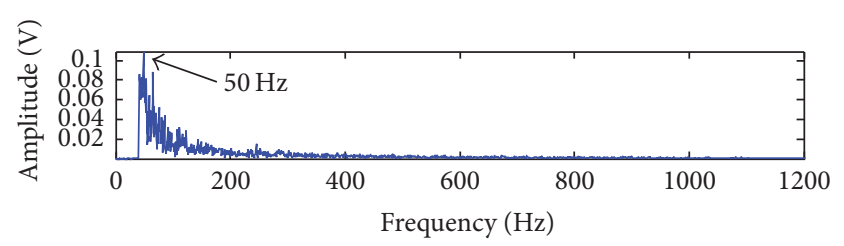

(b)

FIGURE 8: Optimal output signal of IMF2: (a) time-domain waveform; (b) frequency spectrum.

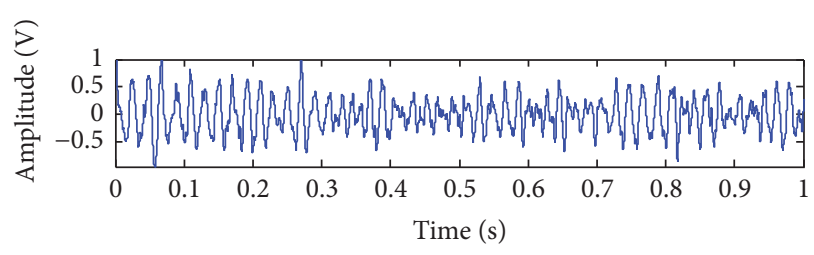

(a)

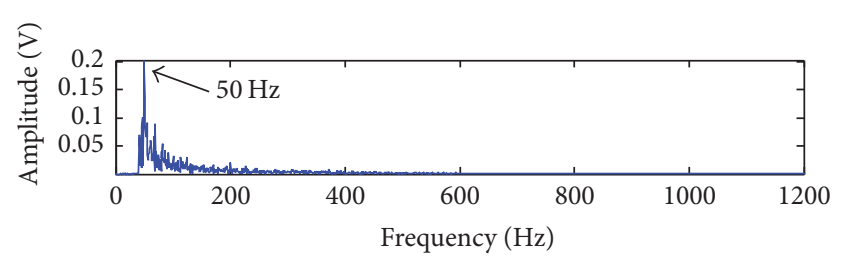

(b)

FIGURE 9: Optimal output signal of IMF3: (a) time-domain waveform; (b) frequency spectrum.

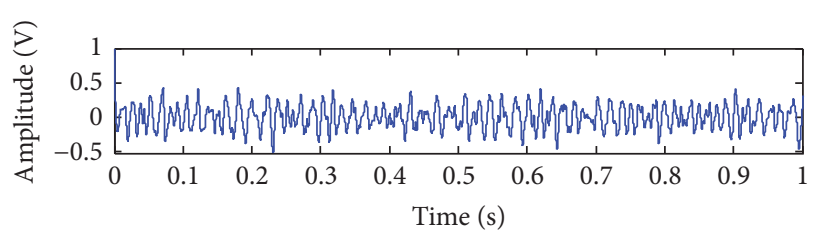

(a)

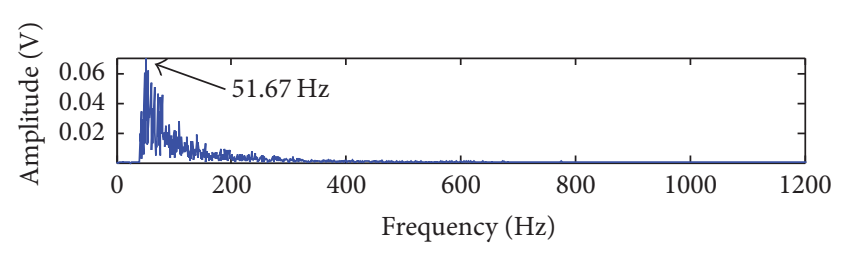

(b)

FIGURE 10: Optimal output signal of IMF4: (a) time-domain waveform; (b) frequency spectrum. 


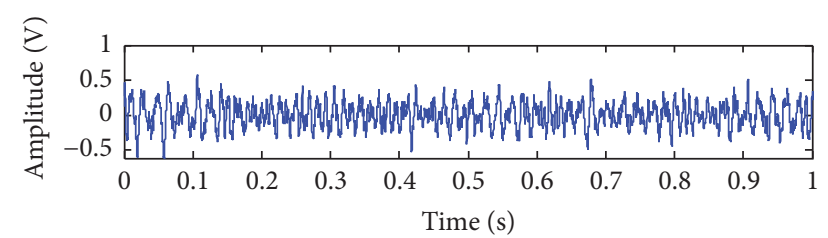

(a)

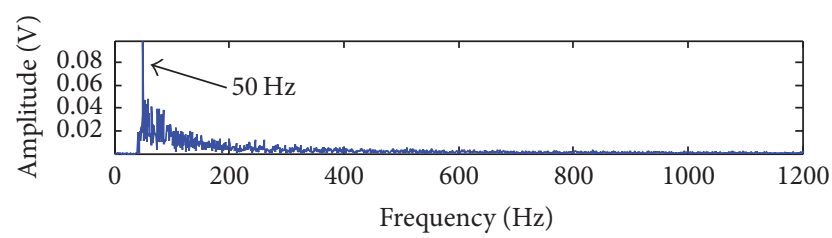

(b)

FIGURE 11: The statistical average of IMFs: (a) time-domain waveform; (b) frequency spectrum.

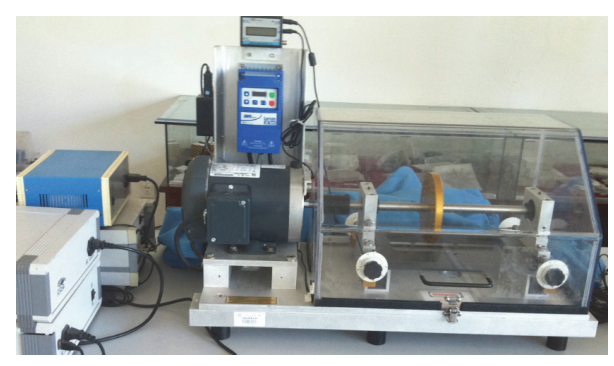

FIGURE 12: Rolling bearing fault test platform.

is $165 \mathrm{~Hz}$ and the variable scale compression rate is 400 . The target frequency is compressed to $(173.25-165) / 400=$ $0.02065 \leq 1 \mathrm{~Hz}$ which meets the requirements of small parameters. Because the actual signal is more complex than the simulation signal, the parameter optimization of stochastic resonance is based on ant colony algorithm. The optimal parameter combinations of adaptive stochastic resonance of IMF1 IMF3 and output signal-to-noise ratio of IMF1 IMF3 are $22.3860,22.2677$, and 22.3304 , respectively, which are an evaluation function in Table 4.

Time-domain and the frequency spectrum of IMF1 IMF3 each in adaptive stochastic resonance are shown in Figures 16 18, respectively.

After adaptive stochastic resonance treatment, the fault frequencies of IMF1 and IMF2 are $170.7 \mathrm{~Hz}$ and $165.3 \mathrm{~Hz}$ which are greatly different from the theoretical value as shown in Figures 16(b) and 17(b). IMF3 is approximate to the characteristic frequency $173.25 \mathrm{~Hz}$ in Figure $18(\mathrm{~b})$, but ambient noise has a large disturbance. From the above analysis, it can be known that even if we reduce the noise by EEMD and the stochastic resonance of each IMF component in the strong background noise, there is still a large deviation between the actual peak and the theoretical peak. The noise interference is still very serious.

In order to reduce noise further, statistical ensemble average on IMF1 IMF3 is performed and the final result is shown in Figure 19.

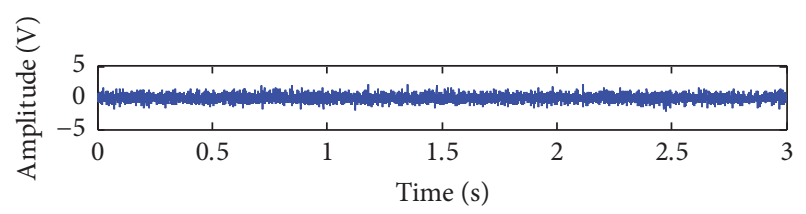

(a)

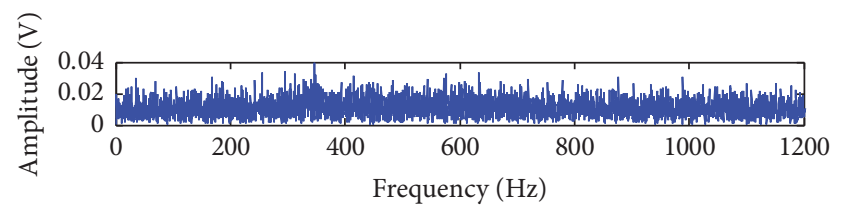

(b)

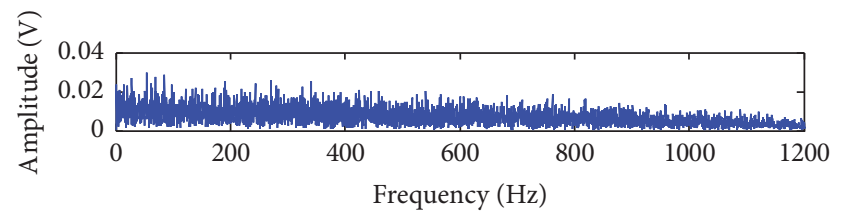

(c)

FIGURE 13: The fault signal of bearing inner race: (a) time-domain waveform; (b) frequency spectrum; (c) envelope spectrum.

TABLE 3: Difference spectrum of the bearing inner race corresponding to IMFs.

\begin{tabular}{lc}
\hline IMF & Difference spectrum \\
\hline IMF1 & 0.4708 \\
IMF2 & 0.4174 \\
IMF3 & 0.9087 \\
IMF4 & 0.07614 \\
IMF5 & 0.01715 \\
IMF6 & 0.02412 \\
IMF7 & 0.0008135 \\
IMF8 & 0.0009225 \\
IMF9 & $3.355 e-6$ \\
IMF10 & 0.001409 \\
IMF11 & 0.001771 \\
IMF12 & 0.0004004 \\
IMF13 & 0.0003416 \\
\hline
\end{tabular}

TABLE 4: The optimal parameter combination of adaptive stochastic resonance of IMF1 IMF3.

\begin{tabular}{lccc}
\hline Parameter & IMF1 & IMF2 & IMF3 \\
\hline$a$ & 0.001 & 0.0003 & 0.012 \\
$b$ & 0.22420 & 0.1618 & 0.0443 \\
Signal-to-noise ratio & 22.3860 & 22.2677 & 22.3304 \\
\hline
\end{tabular}

In Figure 19(b), the maximum spectral peak is $173.7 \mathrm{~Hz}$ (the theoretical value of the bearing inner race is $173.25 \mathrm{~Hz}$ ) and is approximate to the fault characteristic frequency. Compared with Figures 14(b) and 19(b), we cannot see any fault characteristic frequency in Figure 14(b). Thus, the proposed method provides obvious advantages over the EEMD method in extracting weak characteristics from the signal with a 

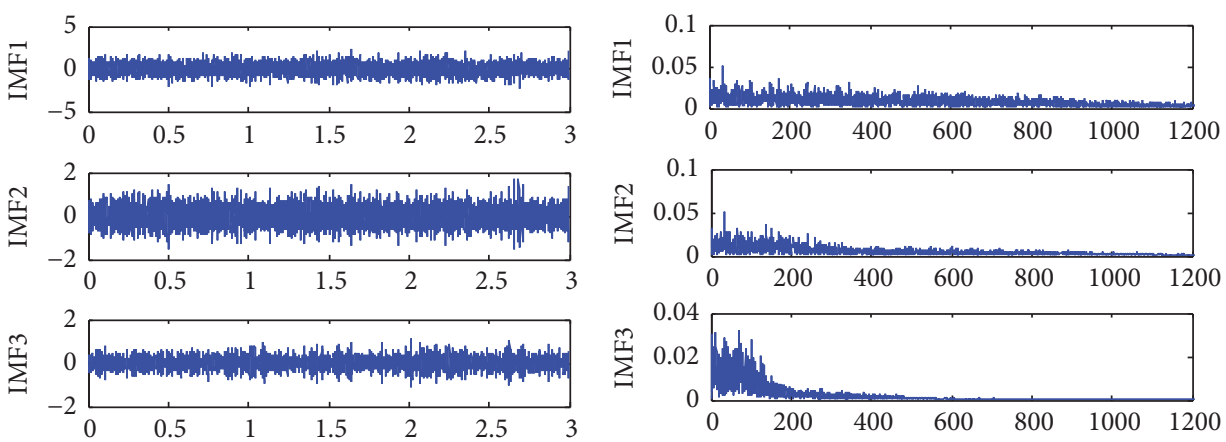

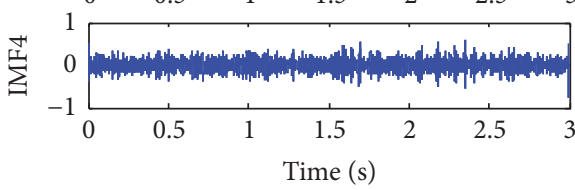

(a)

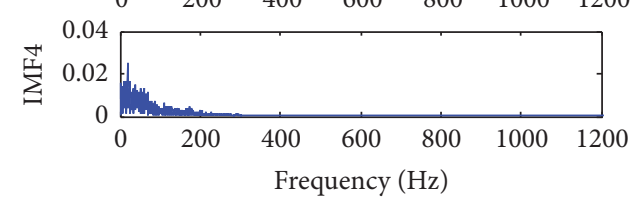

(b)

FIGURE 14: The IMF1 IMF4 of bearing inner race: (a) time-domain waveform; (b) frequency spectrum.

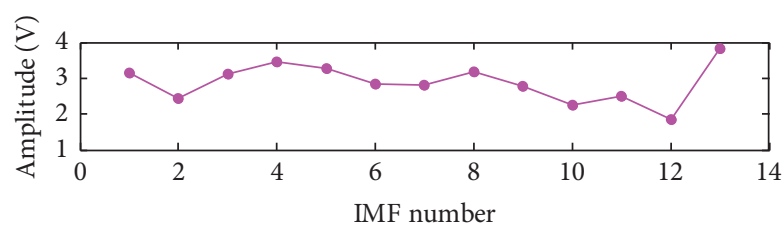

$\longrightarrow$ Kurtosis index

(a)

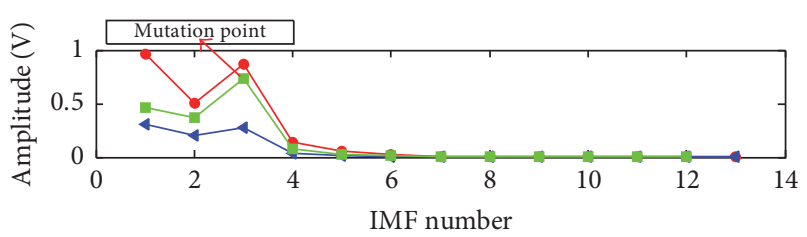

- Weighted kurtosis index

- Cross-correlation coefficient

$\rightarrow-$ Difference spectrum

(b)

FIGURE 15: WKIDS of the inner race of the bearing: (a) kurtosis index; (b) weighted kurtosis index, cross-correlation coefficient, and difference spectrum.

low signal-to-noise ratio. Moreover, the frequency spectra of IMF1, IMF2, and IMF3 each in adaptive stochastic resonance are shown in Figures 16(b), 17(b), and 18(b), respectively; the characteristic frequencies are not the best results. We can assume that the weak fault characteristics cannot also be obtained only by adaptive stochastic resonance. In other words, the proposed method is not only better than EEMD method but also superior to adaptive stochastic resonance.

\section{Conclusions}

In this article, the fault diagnosis methods based on sensitive IMFs selection of EEMD and adaptive stochastic resonance

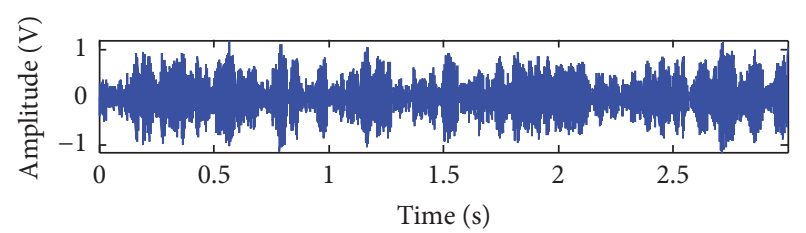

(a)

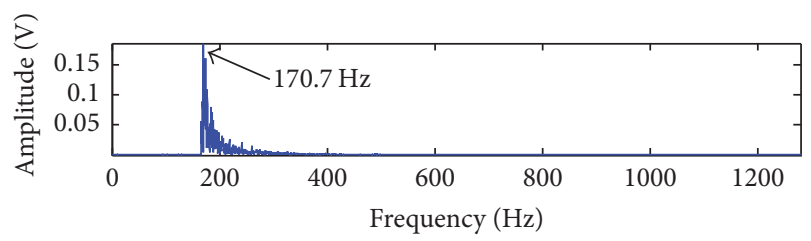

(b)

FIgURE 16: The enhanced result of IMF1 with an inner fault via adaptive stochastic resonance: (a) time-domain waveform; (b) frequency spectrum.

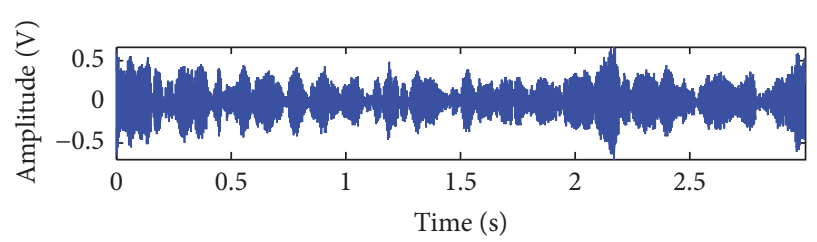

(a)

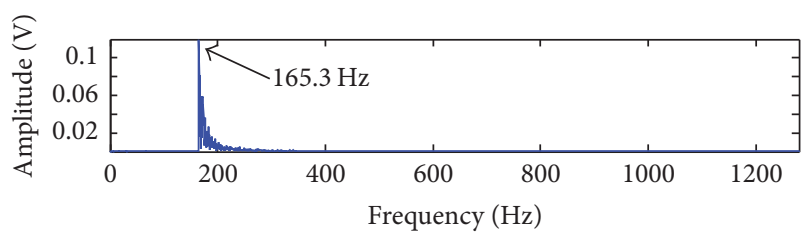

(b)

FIGURE 17: The enhanced result of IMF2 with an inner fault via adaptive stochastic resonance: (a) time-domain waveform; (b) frequency spectrum. 


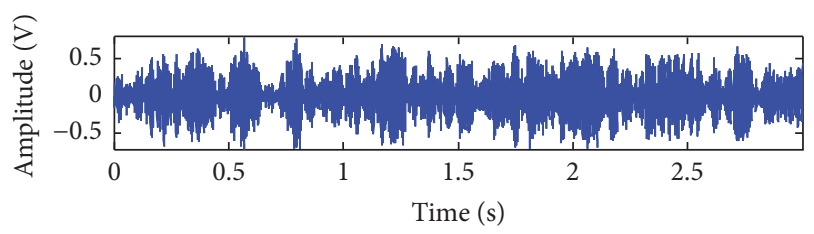

(a)

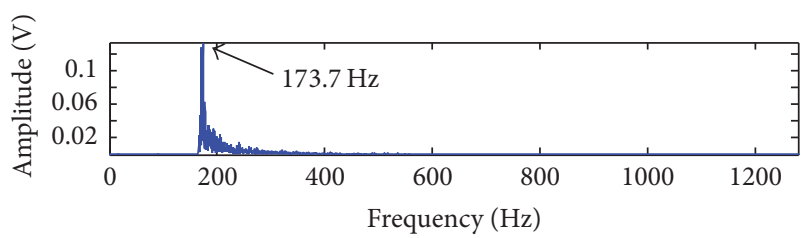

(b)

FIGURE 18: The enhanced result of IMF3 with an inner fault via adaptive stochastic resonance: (a) time-domain waveform; (b) frequency spectrum.

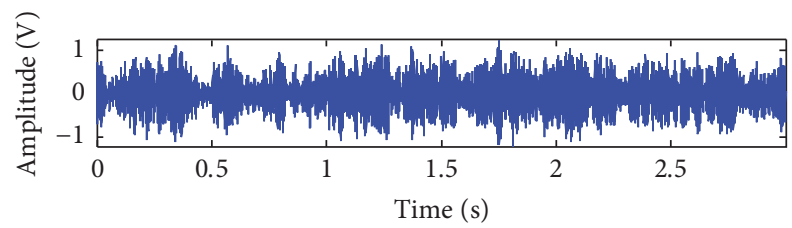

(a)

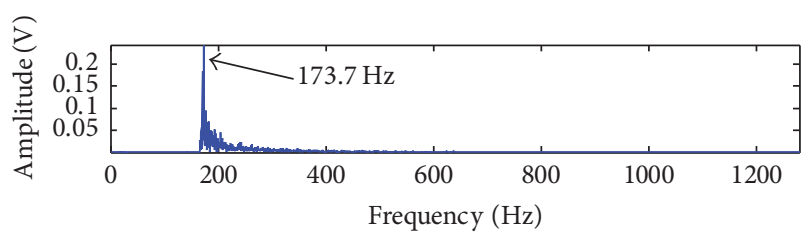

(b)

FIGURE 19: The statistical average of sensitive IMFs: (a) time-domain waveform; (b) frequency spectrum.

are studied, carrying out simulation and bearing experiment, mainly yielding the following conclusions.

(1) A new method of sensitive IMFs selection of EEMD is proposed. WKIDS is constructed by cross-correlation coefficient and kurtosis index. In WKIDS sequence, the maximum point reflects the mutation between the effective IMFs and the useless IMFs. This method can adaptively select the effective IMFs and eliminate the subjectivity of artificial selection IMFs.

(2) Combining effective IMFs selection of EEMD and adaptive stochastic resonance of each effective IMF and ensemble average, the noise of the original signal is greatly reduced and the signal-to-noise ratio is increased. The proposed method detects the weak fault feature accurately and the effect is very obvious.

(3) In the simulation and experiment, compared with EEMD and adaptive stochastic resonance of each sensitive IMF, it is found that EEMD cannot detect the weak fault feature in highly noisy environments. The selected effective IMFs are fed into adaptive stochastic resonance, but the fault characteristic frequency in each enhanced IMF is still disturbed by the noise, and the fault frequency is not obvious. To reduce the noise further, all enhanced effective IMFs are ensemble-averaged. Finally, the weak fault characteristics hidden in the strong noise are extracted. After reducing the noise, adaptive stochastic resonance of the effective IMFs cannot effectively extract the fault, so we can conclude that stochastic resonance only can never detect weak fault characteristics. Hence, the proposed method is better than EEMD and adaptive stochastic resonance.

\section{Competing Interests}

The authors declare that they have no competing interests.

\section{Acknowledgments}

This research is supported by the National Science Foundation of China (51075029).

\section{References}

[1] D. H. Pandya, S. H. Upadhyay, and S. P. Harsha, "Fault diagnosis of rolling element bearing by using multinomial logistic regression and wavelet packet transform," Soft Computing, vol. 18, no. 2, pp. 255-266, 2014.

[2] Q. He, Y. Liu, Q. Long, and J. Wang, "Time-frequency manifold as a signature for machine health diagnosis," IEEE Transactions on Instrumentation and Measurement, vol. 61, no. 5, pp. 12181230, 2012.

[3] B. Hu and B. Li, "Blade crack detection of centrifugal fan using adaptive stochastic resonance," Shock and Vibration, vol. 2015, Article ID 954932, 12 pages, 2015.

[4] Y. Lei, J. Lin, D. Han, and Z. He, "An enhanced stochastic resonance method for weak feature extraction from vibration signals in bearing fault detection," Proceedings of the Institution of Mechanical Engineers, Part C: Journal of Mechanical Engineering Science, vol. 228, no. 5, pp. 815-827, 2014.

[5] A. A. Tabrizi, L. Garibaldi, A. Fasana, and S. Marchesiello, "Performance improvement of ensemble empirical mode decomposition for roller bearings damage detection," Shock and Vibration, vol. 2015, Article ID 964805, 10 pages, 2015.

[6] Z. Wu and N. E. Huang, "Ensemble empirical mode decomposition: a noise-assisted data analysis method," Advances in Adaptive Data Analysis, vol. 1, no. 1, pp. 1-41, 2009.

[7] A. Hu, W. Ma, and G. Tang, "Rolling bearing fault feature extraction method based on ensemble empirical mode decomposition and kurtosis criterion," Proceedings of the Chinese Society of Electrical Engineering, vol. 32, no. 11, pp. 106-111, 2012.

[8] H. Wang, J. Chen, and G. Dong, "Fault diagnosis of rolling bearing's early weak fault based on minimum entropy deconvolution and fast Kurtogram algorithm," Proceedings of the Institution of Mechanical Engineers, Part C: Journal of Mechanical Engineering Science, vol. 229, no. 16, pp. 2890-2907, 2015.

[9] J. Tan, X. Chen, J. Wang et al., "Study of frequency-shifted and re-scaling stochastic resonance and its application to fault diagnosis," Mechanical Systems and Signal Processing, vol. 23, no. 3, pp. 811-822, 2009. 
[10] Y. Xu, J. Li, J. Feng, H. Zhang, W. Xu, and J. Duan, "Lévy noiseinduced stochastic resonance in a bistable system," European Physical Journal B, vol. 86, article 198, 2013.

[11] Q. He, J. Wang, F. Hu, and F. Kong, "Wayside acoustic diagnosis of defective train bearings based on signal resampling and information enhancement," Journal of Sound and Vibration, vol. 332, no. 21, pp. 5635-5649, 2013.

[12] R. Benzi, G. Parisi, A. Sutera et al., "Stochastic resonance in climatic change," Tellus, vol. 34, pp. 10-16, 1982.

[13] L. Zhang, F. Wang, and Z. Hui, "Detecting high frequency weak signal using parameter-adjusted frequency shifting based on Stochastic Resonance (SR)," Physics Procedia, vol. 24, pp. 382389, 2012.

[14] S. Lu, Q. He, H. Zhang, and F. Kong, "Rotating machine fault diagnosis through enhanced stochastic resonance by full-wave signal construction," Mechanical Systems and Signal Processing, vol. 85, pp. 82-97, 2017.

[15] Y. Lei, D. Han, J. Lin, and Z. He, "Planetary gearbox fault diagnosis using an adaptive stochastic resonance method," Mechanical Systems and Signal Processing, vol. 38, no. 1, pp. 113124, 2013.

[16] J. Li, X. Chen, and Z. He, "Adaptive stochastic resonance method for impact signal detection based on sliding window," Mechanical Systems and Signal Processing, vol. 36, no. 2, pp. 240255, 2013.

[17] N. Huang, "The empirical mode decomposition and the Hilbert spectrum for nonlinear and non-stationary time series analysis," Proceedings of the Royal Society of London A, vol. 454, no. 1971, pp. 903-995, 1998.

[18] H. Dong, K. Qi, X. Chen, Y. Zi, Z. He, and B. Li, "Sifting process of EMD and its application in rolling element bearing fault diagnosis," Journal of Mechanical Science and Technology, vol. 23, no. 8, pp. 2000-2007, 2009.

[19] F. Zhao, X. Mei, T. Tao, G. Jiang, and Y. Zhou, "Fault diagnosis of a machine tool rotary axis based on a motor current test and the ensemble empirical mode decomposition method," Proceedings of the Institution of Mechanical Engineers, Part C: Journal of Mechanical Engineering Science, vol. 225, no. 5, pp. 1121-1129, 2011.

[20] Z. Pan, Z. Qiao, and N. Zhang, "Weak signal detection of adaptive stochastic resonance based on shannon entropy of symbolic series," Acta Metrologica Sinica, vol. 36, pp. 496-500, 2015.

[21] Z. Qiao, Y. Lei, J. Lin, and F. Jia, "An adaptive unsaturated bistable stochastic resonance method and its application in mechanical fault diagnosis," Mechanical Systems and Signal Processing, vol. 84, pp. 731-746, 2017.

[22] Q. He and J. Wang, "Effects of multiscale noise tuning on stochastic resonance for weak signal detection," Digital Signal Processing, vol. 22, no. 4, pp. 614-621, 2012.

[23] S. Lu, Q. He, and F. Kong, "Stochastic resonance with WoodsSaxon potential for rolling element bearing fault diagnosis," Mechanical Systems and Signal Processing, vol. 45, no. 2, pp. 488$503,2014$.

[24] Z. Qiao and Z. Pan, "SVD principle analysis and fault diagnosis for bearings based on the correlation coefficient," Measurement Science and Technology, vol. 26, no. 8, Article ID 085014, 2015.

[25] Y. G. Leng, Y. S. Leng, T. Y. Wang, and Y. Guo, "Numerical analysis and engineering application of large parameter stochastic resonance," Journal of Sound and Vibration, vol. 292, no. 3-5, pp. 788-801, 2006.
[26] R. Randall and J. Antoni, "Rolling element bearing diagnostics-a tutorial," Mechanical Systems and Signal Processing, vol. 25, no. 2, pp. 485-520, 2011. 


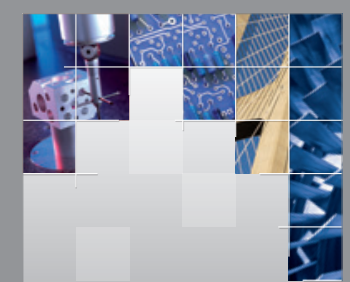

\section{Enfincering}
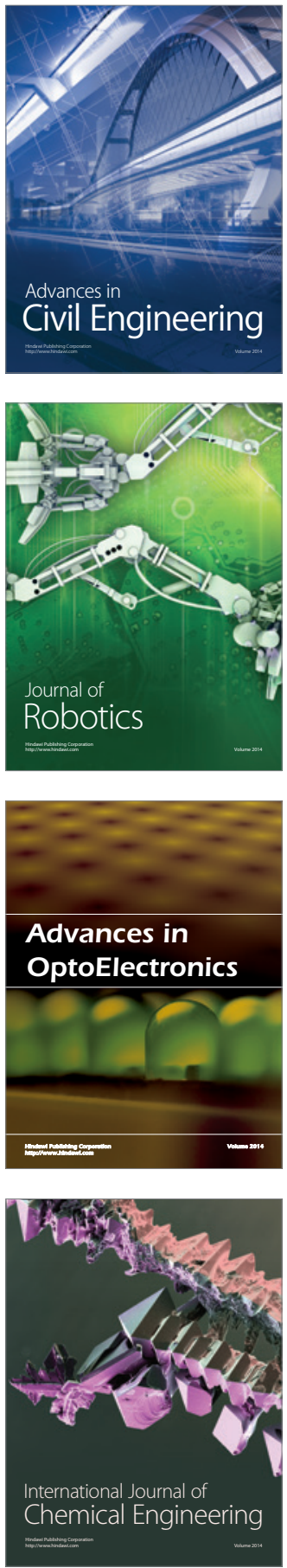

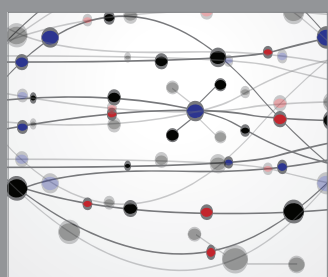

The Scientific World Journal

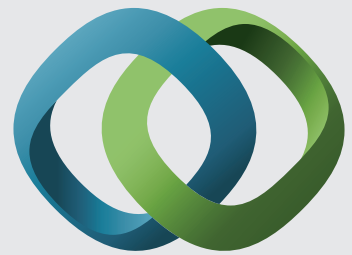

\section{Hindawi}

Submit your manuscripts at

http://www.hindawi.com
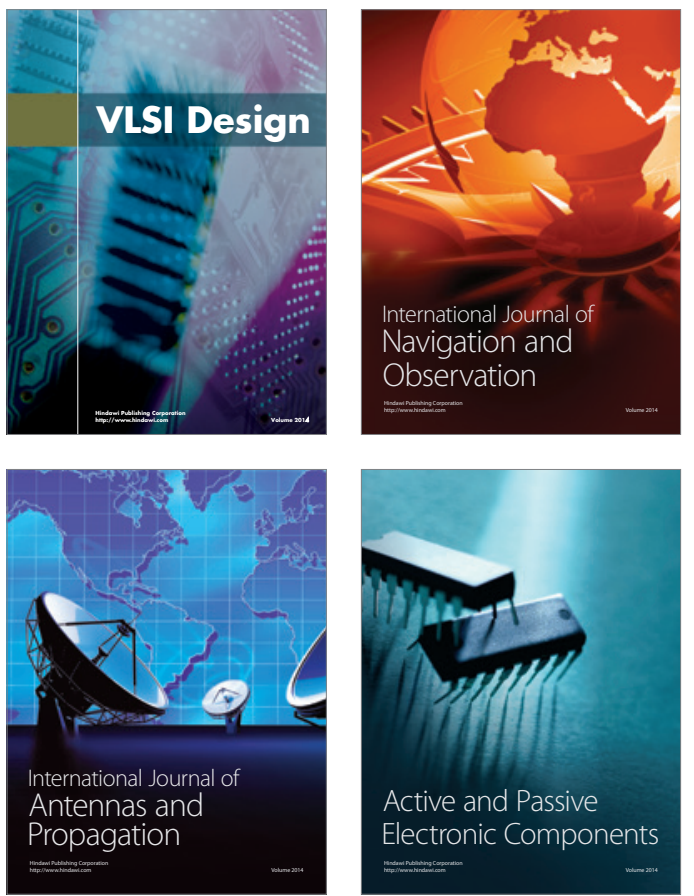
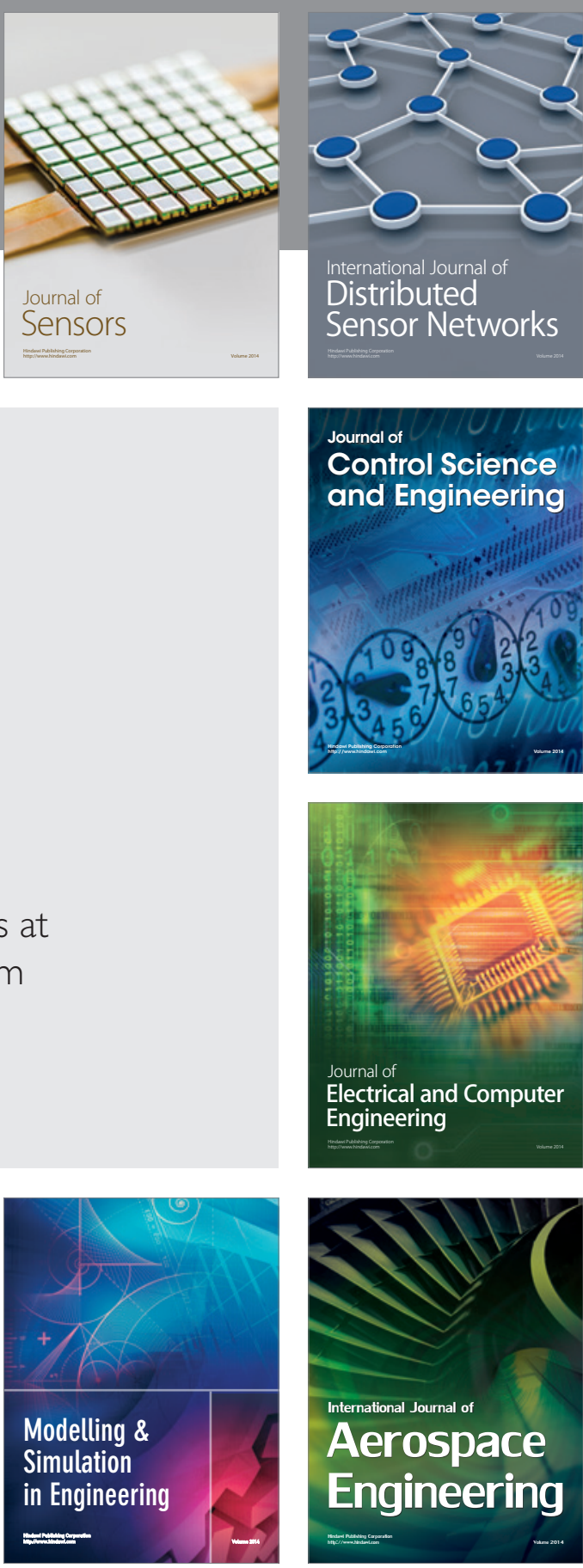

International Journal of

Distributed

Sensor Networks

Journal of

Control Science

and Engineering
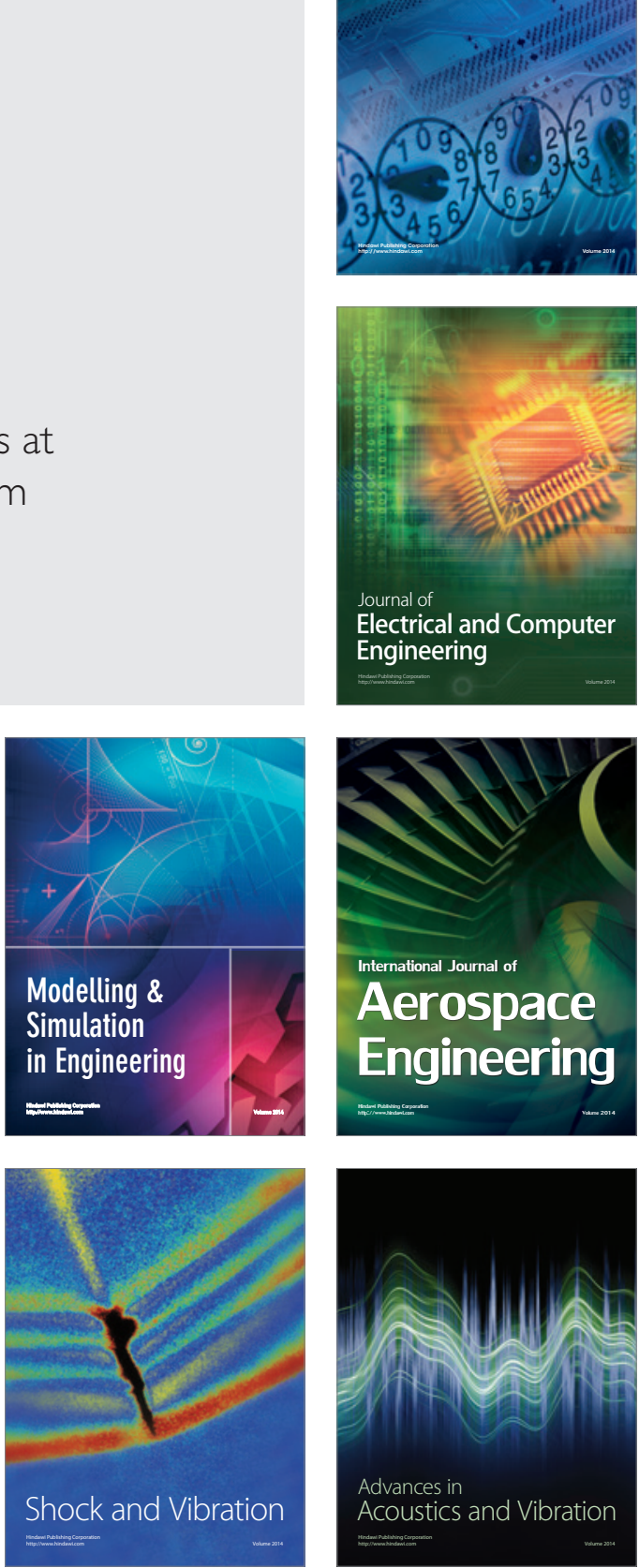